\title{
The Conformal Factor in the SAS Einstein-Maxwell Field Equations and a Central Extension of a Formal Loop Group
}

\author{
Takashi Hashimoto ${ }^{1 \star}$, Ryuichi Sawae ${ }^{2}$ \\ ${ }^{1}$ Department of Mathematics, Faculty of Science, Hiroshima University, Higashi-Hiroshima, \\ 724, Japan. e-mail: r3104@math.sci.hiroshima-u.ac.jp \\ ${ }^{2}$ Department of Management and Information Science, Shikoku University, Tokushima, 771- \\ 11, Japan. e-mail: sawae@ keiei.shikoku-u.ac.jp
}

Received: 22 February 1993/in revised form: 26 April 1993

\begin{abstract}
We consider a relation between the conformal factor in the stationary axisymmetric (SAS) Einstein-Maxwell field equations and a central extension of a formal loop group which is described by a group 2-cocycle on the formal loop group. The corresponding 2-cocycle on the Lie algebra of the formal loop group is the one which describes an affine Lie algebra. As a result, we see that the space of formal solutions with conformal factors is a homogeneous space of a central extension of the Hauser group.
\end{abstract}

\section{Introduction}

In [HS] we have discussed a $\sigma$-model with values in $S(U(1) \times U(2)) \backslash S U(1,2)$ which is derived from the stationary axisymmetric (SAS) Einstein-Maxwell field equations. We formulated the theory of the $\sigma$-model in the category of formal power series by using Takasaki's formal loop group technique [T] and the linearization procedure investigated by Breitenlohner and Maison [BM]. However, we did not incorporate the conformal factor into the theory, neither did we state the homogeneous structure of the space of solutions of the Einstein-Maxwell field equations in stationary axisymmetric space-time.

As to the conformal factor, the second author, in [S], reproduced the results of $[\mathrm{BM}]$ in the category of formal power series and obtained an infinite dimensional homogeneous space structure of the space of formal solutions in the case of the Einstein equations.

In the present paper, following [BM, HS, S], we extend the theory of our $\sigma$-model to the Einstein-Maxwell field equations with $N$ abelian gauge fields in stationary axisymmetric space-times involving the conformal factor. We prove that there is an elegant relation between the conformal factor and a group 2-cocycle on the formal loop group with values in $S U(1, N+1)$, and show that the trivial central

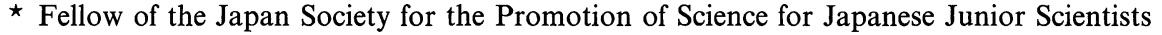


extension of the Hauser group acts transitively on the space of formal solutions of the Einstein-Maxwell field equations with $N$ abelian gauge fields. The corresponding 2-cocycle on the Lie algebra of the formal loop group is the one which describes an affine Lie algebra $[\mathrm{K}]$. This relation was first found by [BM].

Now we derive the equations, which are our starting point, from the stationary axisymmetric Einstein-Maxwell field equations with $N$ abelian gauge potentials.

Let $d s^{2}=g_{\mu v} d x^{\mu} \otimes d x^{\nu}$ be a metric on $\mathbb{R}^{1+3}$ and $\mathbf{A}=\mathbf{A}_{\mu} d x^{\mu}$ an abelian gauge potential with values in $\mathbb{R}^{N}$. Then the Einstein-Maxwell field equations with $N$ abelian gauge fields are given by

$$
R_{\mu \nu}=8 \pi T_{\mu \nu}, \quad \nabla_{\kappa} \mathbf{F}^{\mu \kappa}=0(\mu, v=0,1,2,3),
$$

where $R_{\mu \nu}$ is the Ricci curvature and

$$
\begin{aligned}
\mathbf{F}_{\mu \nu} & =\partial_{\mu} \mathbf{A}_{v}-\partial_{\nu} \mathbf{A}_{\mu}, \\
T_{\mu \nu} & =\frac{1}{4 \pi}\left(\mathbf{F}_{\mu \kappa}{ }^{t} \mathbf{F}_{\nu}{ }^{\kappa}-\frac{1}{4} g_{\mu \nu} \mathbf{F}_{\kappa \iota}{ }^{t} \mathbf{F}^{\mu l}\right) .
\end{aligned}
$$

We adopt the coordinates $\left(x^{0}, x^{1}, x^{2}, x^{3}\right)=(t, \phi, z, \rho)$ with $t$ being time and $(\phi, z, \rho)$ the cylindrical coordinates of $\mathbb{R}^{3}$. Stationary axisymmetric space-times amount to the assumption that a metric is of the form

$$
\begin{aligned}
g & =\left(\begin{array}{rrrr}
h_{00} & h_{01} & & \\
h_{10} & h_{11} & & \\
& & -\lambda & 0 \\
& & 0 & -\lambda
\end{array}\right), \\
\operatorname{det} h & =-\rho^{2},
\end{aligned}
$$

where $\lambda>0, h_{01}=h_{10}$ and $h=\left(h_{i j}\right)$. The field $\lambda$ is called the conformal factor.

For abelian gauge potentials, we fix the gauge so as to $\mathbf{A}_{\mathbf{2}}=\mathbf{A}_{\mathbf{3}}=0$. Since we assume that the fields are stationary and axisymmetric, the functions $h_{i j}$ 's, $\lambda$ and $\mathbf{A}_{i}$ 's depend only on $z$ and $\rho$.

There is still a gauge symmetry that remains after setting $h$ and $\mathbf{A}$ as above, i.e.

$$
h \rightarrow{ }^{t} g h g, \quad \mathbf{A}_{i} \rightarrow \mathbf{A}_{i}+\mathbf{C}_{i}
$$

for $g \in S L(2, \mathbb{R})$ and $\mathbf{C}_{i} \in \mathbb{R}^{N}(i=0,1)$. Therefore, we fix the gauge as follows:

$$
\left.h\right|_{(z, \rho)=(0,0)}=\left(\begin{array}{ll}
1 & 0 \\
0 & 0
\end{array}\right),\left.\quad \mathbf{A}\right|_{(z, \rho)=(0,0)}=0 .
$$

Introducing the Ernst potentials $u \in \mathbb{R}, v \in \mathbb{C}^{N}$ constructed from $h$ and $\mathbf{A}$ by the standard method (cf. [DO][E]), we obtain

Proposition 0.1. The stationary axisymmetric Einstein-Maxwell field equations with $N$ abelian gauge fields are equivalent to the following equations:

$$
\begin{aligned}
& f\left(d * d u+\rho^{-1} d \rho \wedge * d u\right)=\left(d u-2 v^{*} d v\right) \wedge * d u, \\
& f\left(d * d v+\rho^{-1} d \rho \wedge * d v\right)=\left(d u-2 v^{*} d v\right) \wedge * d v,
\end{aligned}
$$




$$
\begin{aligned}
\frac{\partial_{z} \lambda}{\lambda}= & -\frac{\partial_{z} f}{2 f}+\frac{\rho}{2 f^{2}}\left(\partial_{z} f \partial_{\rho} f\right) \\
& -\frac{\rho}{2 f^{2}}\left(\partial_{\rho} u-\partial_{\rho} f-2 v^{*} \partial_{\rho} v\right)\left(\partial_{z} u-\partial_{z} f-2 v^{*} \partial_{z} v\right) \\
& +\frac{\rho}{f}\left(\partial_{z} v^{*} \partial_{\rho} v+\partial_{z} v^{*} \partial_{\rho} v\right), \\
\frac{\partial_{\rho} \lambda}{\lambda}= & -\frac{\partial_{\rho} f}{2 f}+\frac{\rho}{4 f^{2}}\left\{\left(\partial_{\rho} f\right)^{2}-\left(\partial_{z} f\right)^{2}\right\} \\
& +\frac{\rho}{4 f^{2}}\left\{\left(\partial_{z} u-\partial_{z} f-2 v^{*} \partial_{z} v\right)^{2}-\left(\partial_{\rho} u-\partial_{\rho} f-2 v^{*} \partial_{\rho} v\right)^{2}\right\} \\
& -\frac{\rho}{f}\left(\partial_{z} v^{*} \partial_{z} v-\partial_{\rho} v^{*} \partial_{\rho} v\right),
\end{aligned}
$$

where $v^{*}={ }^{t} \bar{v},|v|^{2}=v^{*} v, f=\operatorname{Re} u-|v|^{2}$ and $*$ is the Hodge operator given by $* d z=d \rho, * d \rho=-d z$.

The first two equations are called the Ernst equations.

Corresponding to the gauge fixing (0.1), we shall consider the solutions under the conditions

$$
\left.u\right|_{(z, \rho)}=(0,0)=1 \text { and }\left.v\right|_{(z, \rho)=(0,0)}=0 .
$$

It is essential to introduce the function $\tau=f^{1 / 2} \lambda$ and we shall consider $\tau$, instead of $\lambda$, throughout the paper.

\section{Ernst Equation}

Let $\theta$ be Cartan involution of $G L(N+2, \mathbb{C})$ defined by $g \mapsto g^{*-1}$ and $G$ a subgroup of $G L(N+2, \mathbb{C})$ defined by

$$
\left\{g \in G L(N+2, \mathbb{C}) ; g^{*} J g=J, \operatorname{det} g=1\right\},
$$

where $J=\left(\begin{array}{ccc} & 1_{N} & \end{array}\right)$ and $1_{N}$ denotes the $N \times N$ identity matrix. Note that $G$ is isomorphic to $S U(1, N+1)$. Let $K$ be the subgroup of $G$ such that each element of $K$ is fixed by $\theta$. Then $K$ is a maximal subgroup of $G$.

Let $\mathfrak{g}$ and $\mathfrak{f}$ be the Lie algebras of $G$ and $K$, respectively. Then $\mathfrak{g}$ decomposes as

$$
\mathfrak{g}=\mathfrak{l} \oplus \mathfrak{p},
$$

where $\mathfrak{f}=\{x \in \mathfrak{g} ; \theta X=X\}$ and $\mathfrak{p}=\{x \in \mathfrak{g} ; \theta X=-X\}$ with $\theta$ the Cartan involution of $g$ induced from $\theta$ of $G$. 
We fix subgroups $A$ and $N$ of $G$ as follows:

$$
\begin{aligned}
& A=\left\{\left(\begin{array}{ccc}
a & & \\
& 1_{N} & \\
& & 1 / a
\end{array}\right) ; a>0\right\}, \\
& N=\left\{\left(\begin{array}{ccc}
1 & \\
v & 1_{N} \\
x+i|v|^{2} / 2 & i v^{*} & 1
\end{array}\right) ; x \in \mathbb{R}, v \in \mathbb{C}^{N}\right\},
\end{aligned}
$$

where $|v|^{2}=v^{*} v$. Then we have $G=K A N$ (Iwasawa decomposition).

Let $R$ be a ring of formal power series in $z$ and $\rho$ over $\mathbb{C}$ i.e. $R=\mathbb{C}[[z, \rho]]$. We regard $z$ and $\rho$ as real variables, which means, $\bar{z}=z$ and $\bar{\rho}=\rho$. We denote by $*$ the anti-involution of $\mathfrak{g l}(N+2, R)=\mathfrak{g l}(N+2, \mathbb{C}) \otimes_{\mathbb{C}} R$ which is an obvious extension of the canonical anti-involution * of $\operatorname{gl}(N+2, \mathbb{C})$ and by $\theta_{R}$ an involution of $G L(N+2, R)$ defined by $\theta_{R}(g)=g^{*-1}$ for $g \in G L(N+2, R)$. Let $G_{R}$ be a subgroup of $G L(N+2, R)$ defined by

$$
\left\{g \in G L(N+2, R) ; g^{*} J g=J, \operatorname{det} g=1\right\} .
$$

Then, corresponding to $G=K A N, G_{R}$ decomposes as $G_{R}=K_{R} A_{R} N_{R}$, where $K_{R}$, $A_{R}$ and $N_{R}$ denote subgroups of $G_{R}$ consisting of matrices with values in $K, A$ and $N$ respectively, each of whose components is an element of $R$.

Now we parametrize an element of $A_{R} N_{R}$ as follows:

$$
P=\left(\begin{array}{ccc}
f^{1 / 2} & 0 & 0 \\
\sqrt{2 v} & 1_{N} & 0 \\
\left(\psi+i|v|^{2}\right) / f^{1 / 2} & \sqrt{2} i v^{*} / f^{1 / 2} & f^{-1 / 2}
\end{array}\right),
$$

where $f$ and $v$ are the same ones as in (0.2) and (0.3), and $\psi=\operatorname{Im} u$.

The following fact is well known.

Proposition 1.1. Under the parametrization of (1.2), we put $M=\theta_{R}\left(P^{-1}\right) P$. Then the Ernst equations $(0.2)$ and $(0.3)$ are equivalent to the following equation:

$$
d\left(\rho * d M M^{-1}\right)=0 .
$$

Moreover the function $\tau$ is a solution of $(0.4)$ and $(0.5)$ if and only if it is a solution of the following equations:

$$
\begin{aligned}
\tau^{-1} \partial_{z} \tau & =\frac{\rho}{4} \operatorname{tr}\left(\partial_{z} M M^{-1} \partial_{\rho} M M^{-1}\right) \\
\tau^{-1} \partial_{\rho} \tau & =\frac{\rho}{8} \operatorname{tr}\left(\left(\partial_{\rho} M M^{-1}\right)^{2}-\left(\partial_{z} M M^{-1}\right)^{2}\right) .
\end{aligned}
$$

The integrability of $\tau$ follows easily from (1.4) and (1.5). Equation (1.3) is also called the Ernst equation. We shall consider the solutions satisfying

$$
\left.P\right|_{(z, \rho)=(0,0)}=1,
$$

which corresponds to the gauge fixing condition (0.6). 
We denote by $B$ the real part of the trace form on $\mathfrak{g l}(N+2, R)$ :

$$
B(X, Y)=\operatorname{Re} \operatorname{tr}(X Y) \text { for } X, Y \in \mathfrak{g l}(N+2, R) .
$$

The Lie algebras $\mathfrak{g}_{R}$ of $G_{R}$ and $\mathfrak{f}_{R}$ of $K_{R}$ can be identified with $\mathfrak{g} \otimes_{\mathbb{R}} R$ and $\mathfrak{f} \otimes_{\mathbb{R}} R$, respectively. Note that $\mathfrak{f}_{R}$ and $\mathfrak{p}_{R}=\left\{X \in \mathfrak{g}_{R} ; \theta_{R} X=-X\right\} \cong \mathfrak{p} \otimes_{\mathbb{R}} R$ are orthogonal to each other with respect to $B$, where $\theta_{R}$ also denotes the involution of $\mathfrak{g}_{R}$ induced from that of $G_{R}$.

It is also known that Eq. (1.3) can be rewritten as the integrability condition of a 1 -form with values in $\mathfrak{g}$ each of whose component is an element of $\mathbb{C}(z, \rho) \otimes_{\mathbb{C}} \mathbb{C}[[t]]$, where $\mathbb{C}(z, \rho)$ is the quotient field of $R=\mathbb{C}[[z, \rho]]$ and $t$ an indeterminate called "spectral parameter." Namely, let $\mathscr{A}$ and $\mathscr{I}$ be 1 -forms defined by

$$
\mathscr{A}=\frac{1}{2}\left(d P P^{-1}+\theta_{R}\left(d P P^{-1}\right)\right), \quad \mathscr{I}=\frac{1}{2}\left(d P P^{-1}-\theta_{R}\left(d P P^{-1}\right)\right)
$$

for any $P \in A_{R} N_{R}$, and put

$$
\Omega_{P}=\mathscr{A}+\left(\frac{1-t^{2}}{1+t^{2}}-\frac{2 t}{1+t^{2}} *\right) \mathscr{I},
$$

where $*$ is the Hodge operator given by $* d z=d \rho, * d \rho=-d z$. We extend the canonical exterior derivative $d$ on $\mathbb{C}(z, \rho)$ to that on $\mathbb{C}(z, \rho) \otimes_{\mathbb{C}} \mathbb{C}[[t]]$ by defining

$$
d t=\frac{t}{\left(1+t^{2}\right) \rho}\left(\left(1-t^{2}\right) d \rho+2 t d z\right) .
$$

Note then that $d^{2} t=0$. Now we have

Proposition 1.2. $\Omega_{P}$ satisfies the integrability condition, i.e.,

$$
d \Omega_{P}-\Omega_{P} \wedge \Omega_{P}=0
$$

if and only if $P$ is a solution of (1.3).

This can be proved straightforward, using (1.6).

Moreover, let $\mathscr{I}=\mathscr{I}_{z} d z+\mathscr{I}_{\rho} d \rho$. Then Eqs. (1.4) and (1.5) can be written in terms of $\mathscr{I}_{z}$ and $\mathscr{I}_{\rho}$ as

$$
\begin{aligned}
\tau^{-1} \partial_{z} \tau & =\rho B\left(\mathscr{I}_{z}, \mathscr{I}_{\rho}\right), \\
\tau^{-1} \partial_{\rho} \tau & =\frac{\rho}{2}\left(B\left(\mathscr{I}_{\rho}, \mathscr{I}_{\rho}\right)-B\left(\mathscr{I}_{z}, \mathscr{I}_{z}\right)\right) .
\end{aligned}
$$

It follows from Proposition 1.2 that if $P$ is a solution of the Ernst equation, then there exists a potential $p=\sum_{n \geqq 0} p_{n} t^{n}$ such that each entry of $p_{n}$ is an element of $\mathbb{C}(z, \rho)$ and

$$
d p=\Omega_{P} \cdot p \quad \text { and } p_{0}=P .
$$

\section{Hauser Group}

We introduce formal loop algebras and formal loop groups, following [T].

Put $F_{0}=R=\mathbb{C}[[z, \rho]]$ and $F_{n}=\rho^{|n|} R$ for a nonzero integer $n$. We introduce a topology in $R$ by declaring that $\left\{F_{n}\right\}_{n \geqq 0}$ forms a fundamental neighborhoods system of 0 . Note that $F_{m} F_{n} \subset F_{m+n}$ for $m, n \geqq 0$. 
Then we define a formal loop algebra $\mathscr{F} \mathrm{gl}$ by

$$
\mathscr{F} \mathfrak{g l}=\left\{X=\sum_{n \in \mathbb{Z}} X_{n} t^{n} ; X_{n} \in \mathfrak{g l}\left(N+2, F_{n}\right)\right\} .
$$

Let $*$ be an anti-involution of $\mathscr{F} \mathfrak{g l}$ defined by

$$
X^{*}=\sum_{n \in \mathbb{Z}} X_{n}^{*}(-1 / t)^{n}
$$

for $X=\sum_{n \in \mathbb{Z}} X_{n} t^{n}$, where the anti-involution * in the right-hand side is the one of $\mathfrak{g l}(N+2, R)$ given in Sect. 1 . This is well-defined by the definition of our filtration $\left\{F_{n}\right\}_{n \in \mathbb{Z}}$.

Remark that $\Omega_{P}$ with $P \in A_{R} N_{R}$ is not an element of $\mathscr{F} \mathrm{gl}$, however, we can define $\Omega_{P}^{*}$ by

$$
\Omega_{P}^{*}=\mathscr{A}^{*}+\left(\frac{1-(-1 / t)^{2}}{1+(-1 / t)^{2}}-\frac{2(-1 / t)}{1+(-1 / t)^{2}} *\right) \mathscr{I}^{*},
$$

where * in the right-hand side is the anti-involution of $\mathfrak{g l}(N+2, R)$. Then it follows immediately that

$$
\Omega_{P}^{*}=-\Omega_{P} \quad \text { for } P \in A_{R} N_{R}
$$

We define a formal loop group $\mathscr{F} G L$, following $[\mathrm{T}]$, by

$$
\mathscr{F} G L=\left\{g=\sum_{n \in \mathbb{Z}} g_{n} t^{n} ; g_{n} \in \mathfrak{g l}\left(N+2, F_{n}\right), g_{0} \text { is invertible }\right\} .
$$

Since $\mathscr{F} G L$ is canonically embedded in $\mathscr{F} \mathrm{gl}$, we can define an involution $\theta^{(\infty)}$ of $\mathscr{F} G L$ by

$$
\theta^{(\infty)}(g)=\left(g^{*}\right)^{-1} \quad \text { for } g \in \mathscr{F} G L
$$

which we call Cartan involution of $\mathscr{F} G L$.

Define subgroups of $\mathscr{F} G L$ as follows:

$$
\begin{aligned}
& \mathscr{F} \mathscr{G}=\left\{g=\sum_{n \in \mathbb{Z}} g_{n} t^{n} \in \mathscr{F} G L ; g^{*} J g=J, \operatorname{det} g=1\right\}, \\
& \mathscr{F} \mathscr{G}_{0}=\left\{g=\sum_{n \in \mathbb{Z}} g_{n} t^{n} \in \mathscr{F} \mathscr{G} ;\left.g_{0}\right|_{(z, \rho)=(0,0)}=1\right\}, \\
& \mathscr{F} \mathscr{K}=\left\{k=\sum_{n \in \mathbb{Z}} k_{n} t^{n} \in \mathscr{F} \mathscr{G} ; \theta^{(\infty)} k=k\right\}, \\
& \mathscr{F} \mathscr{P}=\left\{p=\sum_{n \in \mathbb{Z}} p_{n} t^{n} \in \mathscr{F} \mathscr{G} ; p_{0} \in A_{R} N_{R}, p_{n}=0 \text { if } n<0\right\} .
\end{aligned}
$$

Then, using the Birkhoff decomposition ((3.17), [T]), we can decompose uniquely an element $g \in \mathscr{F} \mathscr{G}$ as

$$
g=k p \quad(k \in \mathscr{F} \mathscr{K}, p \in \mathscr{F} \mathscr{P})
$$


Let $s$ be another indeterminate. Define an infinite dimensional group $\mathscr{G}^{(\infty)}$, which we call Hauser group, by

$$
\mathscr{G}^{(\infty)}=\left\{g=\sum_{n \geqq 0} g_{n} s^{n} \in G L(N+2, \mathbb{C}[[s]]) ; g^{*} J g=J, \operatorname{det} g=1, g_{0}=1\right\},
$$

where $\mathbb{C}[[s]]$ is a ring of formal power series in $s$ over $\mathbb{C}$ and $g^{*}=\sum g_{n}^{*} s^{n}$.

Let $j$ be a homomorphism of $G L(N+2, \mathbb{C}[[s]])$ into $\mathscr{F} G L$ given by

$$
j: g=\sum_{n \geqq 0} g_{n} s^{n} \mapsto j(g)=\sum_{n \geqq 0} g_{n}\left(\rho\left(\frac{1}{t}-t\right)+2 z\right)^{n} .
$$

Then it is easy to see that $j$ is injective and that the image of $\mathscr{G}^{(\infty)}$ by $j$ is in $\mathscr{F} \mathscr{G}_{0}$. We denote by $\mathscr{F} \mathscr{H}$ the image of $\mathscr{G}^{(\infty)}$ by $j$. The following equations characterize the elements of $\mathscr{F} \mathscr{H}$ in $\mathscr{F} \mathscr{G}$.

Lemma 2.1. An element $g \in \mathscr{F} \mathscr{G}$ belongs to $\mathscr{F} \mathscr{H}$ if and only if $g$ satisfies the following equations:

$$
\begin{aligned}
& \partial_{t} g=-\rho\left(\partial_{z}+\frac{1}{t} \partial_{\rho}\right) g, \\
& \partial_{t} g=-\frac{\rho}{2}\left(1+\frac{1}{t^{2}}\right) \partial_{z} g .
\end{aligned}
$$

This characterization will play an important role in the proof of our main theorem. For proof, we refer to [S].

Definition. Let $\mathscr{F} \mathscr{P}$ be as in (2.7). We define $\mathscr{S P P}$ to be a subset of $\mathscr{F} \mathscr{P}$ consisting of elements $p=\sum_{n \geqq 0} p_{n} t^{n}$ which satisfy the following conditions:

$$
d p=\Omega_{p_{0}} \cdot p \text { and }\left.p_{0}\right|_{(z, \rho)=(0,0)}=1 .
$$

We call $\mathscr{S} \mathscr{P}$ the space of potentials.

It follows from (2.11) that $p_{0}$ is a solution of the Ernst equation (1.3) for $p=\sum_{n \geqq 0} p_{n} t^{n} \in \mathscr{S} \mathscr{P}$. Equation (2.11) is equivalent to the following equations:

$$
\begin{aligned}
& \partial_{z} p+\frac{2 t^{2}}{\left(1+t^{2}\right) \rho} \partial_{t} p=\Omega_{1} p \\
& \partial_{\rho} p+\frac{t\left(1-t^{2}\right)}{\left(1+t^{2}\right) \rho} \partial_{t} p=\Omega_{2} p,
\end{aligned}
$$

where we define $\Omega_{1}$ and $\Omega_{2}$ by $\Omega_{p_{0}}=\Omega_{1} d z+\Omega_{2} d \rho$.

Put

$$
D_{1}=\partial_{z}+\frac{2 t^{2}}{\left(1+t^{2}\right) \rho} \partial_{t} \quad \text { and } D_{2}=\partial_{\rho}+\frac{t\left(1-t^{2}\right)}{\left(1+t^{2}\right) \rho} \partial_{t}
$$

for brevity.

Theorem 2.2. An element $p \in \mathscr{S} \mathscr{P}$ satisfies

$$
\begin{aligned}
& \partial_{t}\left(p^{*} p\right)=-\rho\left(\partial_{z}+\frac{1}{t} \partial_{\rho}\right)\left(p^{*} p\right), \\
& \partial_{t}\left(p^{*} p\right)=-\frac{\rho}{2}\left(1+\frac{1}{t^{2}}\right) \partial_{z}\left(p^{*} p\right) .
\end{aligned}
$$


Conversely, if $p \in \mathscr{F} \mathscr{P} \cap \mathscr{F} \mathscr{G}_{0}$ satisfies Eqs. (2.13.a) and (2.13.b), then $p$ belongs to $\mathscr{S} \mathscr{P}$, namely, it satisfies Eqs. (2.12.a) and (2.12.b).

Proof. It can be checked by direct calculation that (2.13.a) and (2.13.b) are equivalent to $D_{1}\left(p^{*} p\right)=0$ and $D_{2}\left(p^{*} p\right)=0$. But then, we have, for $p \in \mathscr{S P}$,

$$
\begin{aligned}
D_{1}\left(p^{*} p\right) & =D_{1} p^{*} p+p^{*} D_{1} p \\
& =\left(\Omega_{1} p\right)^{*} p+p^{*}\left(\Omega_{1} p\right) \\
& =-p^{*} \Omega_{1} p+p^{*} \Omega_{1} p \\
& =0,
\end{aligned}
$$

since $\Omega_{1}^{*}=-\Omega_{1}$. Similarly, we can show that $D_{2}\left(p^{*} p\right)=0$.

Conversely, let $p \in \mathscr{F} \mathscr{P}$ satisfy (2.13.a) and (2.13.b). Then we have

$$
\begin{gathered}
-\left(\partial_{t} p p^{-1}\right)^{*}-\rho\left(\left(\partial_{z} p p^{-1}\right)^{*}+\frac{1}{t}\left(\partial_{\rho} p p^{-1}\right)^{*}\right) \\
=\partial_{t} p p^{-1}+\rho\left(\partial_{z} p p^{-1}+\frac{1}{t} \partial_{\rho} p p^{-1}\right) \\
-\left(\partial_{t} p p^{-1}\right)^{*}-\frac{\rho}{2}\left(1+\frac{1}{t^{2}}\right)\left(\partial_{z} p p^{-1}\right)^{*}=\partial_{t} p p^{-1}+\frac{\rho}{2}\left(1+\frac{1}{t^{2}}\right) \partial_{z} p p^{-1} .
\end{gathered}
$$

In (2.14) the left-hand side contains only the terms of $t^{n}(n \leqq 0)$, while the right-hand side those of $t^{n}(n \geqq-1)$, which implies that the coefficients of $t^{n}(n \leqq-2)$ in the 1.h.s. vanish. Therefore, we obtain

$$
\partial_{t} p p^{-1}+\rho\left(\partial_{z} p p^{-1}+\frac{1}{t} \partial_{\rho} p p^{-1}\right)=\rho\left(\Omega_{1}+\frac{1}{t} \Omega_{2}\right),
$$

since the coefficient of $t^{-1}$ in the 1.h.s. of (2.14) is equal to $\rho \partial_{\rho} p_{0} p_{0}^{-1}\left(=\right.$ coeff. of $t^{-1}$ in the r.h.s. of (2.14)). Note that, comparing the coefficients of $t^{-1}$ in both sides of (2.14), we obtain

$$
\partial_{\rho} p_{0} p_{0}^{-1}+\left(\partial_{\rho} p_{0} p_{0}^{-1}\right)^{*}=\text { coeff. of } t^{-1} \text { in }\left(\partial_{z} p p^{-1}\right)^{*} .
$$

Similarly, the 1.h.s. of (2.15) contains only the terms of $t^{n}(n \leqq 0)$, while the r.h.s. those of $t^{n}(n \geqq-2)$, which implies that the coefficients of $t^{n}(n \leqq-3)$ in the 1.h.s. vanish. Therefore, we obtain

$$
\partial_{t} p p^{-1}+\frac{\rho}{2}\left(1+\frac{1}{t^{2}}\right) \partial_{z} p p^{-1}=\frac{\rho}{2}\left(1+\frac{1}{t^{2}}\right) \Omega_{1}
$$

where we used (2.17).

Now it is easy to see that (2.16) and (2.18) are equivalent to (2.12.a) and (2.12.b). Let $p \in \mathscr{S} \mathscr{P}$ and $g \in \mathscr{G}^{(\infty)}$. By (2.8) there exist $k \in \mathscr{F} \mathscr{K}$ and $p_{g} \in \mathscr{F} \mathscr{P}$ such that

$$
p \cdot j(g)=k^{-1} \cdot p_{g} .
$$

Then, it follows immediately from Theorem 2.2 that $p_{g}$ is in $\mathscr{S} \mathscr{P}$. Thus we can define an action of the Hauser group $\mathscr{G}^{(\infty)}$ on $\mathscr{S} \mathscr{P}$ to the right by

$$
\mathscr{S} \mathscr{P} \times \mathscr{G}^{(\infty)} \rightarrow \mathscr{S} \mathscr{P} \quad(p, g) \mapsto p_{g},
$$

where $p_{g}$ is given by $(2.19)$. 
From the fact that an element $g=\sum_{n \geqq 0} g_{n} s^{n} \in \mathscr{G}^{(\infty)}$ such that $g^{*}=g$ and such that $g_{0}$ is positive definite decomposes as $g=h^{*} h$ for some $h \in \mathscr{G}^{(\infty)}$, we have

Corollary 2.3. The action of $\mathscr{G}^{(\infty)}$ on $\mathscr{S P}$ given by $(2.20)$ is transitive.

Remark. As we mentioned in [S], our group $\mathscr{G}^{(\infty)}$ is too small to obtain all solutions of the Ernst equation (1.3) through the action (2.20).

\section{2-Cocycle on $\mathscr{F} \mathscr{G}_{0}$}

The formal loop algebra $\mathscr{F}$ gl becomes a Lie algebra with Lie bracket $[X, Y]=X Y-Y X$. The map

$$
\text { exp: } \mathscr{F} \mathfrak{g l} \rightarrow \mathscr{F} G L
$$

given by

$$
\exp X=e^{X}=\sum_{n \geqq 0} \frac{X^{n}}{n !}
$$

is called the formal exponential map. Note that for any $g \in \mathscr{F} \mathscr{G}_{0}$ we can find a unique element $X$ in $\mathscr{F} \mathrm{gl}$ such that $g=e^{X}$, since the logarithm given by

$$
\log (1+A)=\sum_{n \geqq 1} \frac{(-1)^{n-1}}{n} A^{n}
$$

is well-defined and satisfies

$$
e^{\log (1+A)}=1+A
$$

for $A=\sum_{n \in \mathbb{Z}} a_{n} t^{n} \in \mathscr{F} \mathrm{gl}$ with $a_{0} \in \mathfrak{g l}(N+2, \mathrm{~m})$, where $\mathrm{m} \subset R$ is the maximal ideal.

For $X, Y$ in $\mathscr{F}$ gl, let $c_{n}(X, Y)(n=1,2, \ldots)$ be the elements in $\mathscr{F} \mathrm{gl}$ which are determined by

$$
\exp v X \exp v Y=\exp \sum_{n \geqq 0} c_{n}(X, Y) v^{n},
$$

where $v$ is an indeterminate. Furthermore $c_{n}$ 's are uniquely determined by the following recursion formulas (see [V]):

$$
\begin{aligned}
c_{1}(X, Y)=X+Y \\
(n+1) c_{n+1}(X, Y)=\frac{1}{2}\left[X-Y, c_{n}(X, Y)\right] \\
+\sum_{p \geqq 1,2 p \leqq n} K_{2 p} \sum_{\substack{k_{1}, \ldots, k_{2 p}>0 \\
k_{1}+}}\left[c_{k_{1}}(X, Y),\left[\ldots,\left[c_{k_{2 p}=n}(X, Y), X+Y\right] \ldots\right](n \geqq 1),\right.
\end{aligned}
$$

where $K_{2 p}$ 's are determined by

$$
\frac{x}{1-e^{-x}}-\frac{1}{2} x=1+\sum_{p \geqq 1} K_{2 p} x^{2 p} .
$$

We set $C(X, Y)=\sum_{n \geqq 1} c_{n}(X, Y)$. Then $C(X, Y)$ is a well-defined element of $\mathscr{F} \mathrm{gl}$ for $X, Y$ such that $X_{0}, Y_{0} \in \mathfrak{g l}(N+2, \mathrm{~m})$. 
Lemma 3.1. For $n \geqq 2$, there exists a $\mathscr{F}$ gl-valued function $L_{n}(\cdot, \cdot)$ which satisfies

$$
c_{n}(X, Y)=\left[X, L_{n}(X, Y)\right]+\left[Y, L_{n}(-Y,-X)\right]
$$

for $X, Y \in \mathscr{F} \mathrm{gl}$.

Proof. It is easy to see that $c_{n}$ can be written as

$$
c_{n}(X, Y)=\left[X, A_{n}(X, Y)\right]+\left[Y, B_{n}(X, Y)\right] .
$$

Let $X, Y \in \mathscr{F}$ gl. Then, since $c_{n}(X, Y)=-c_{n}(-Y,-X)$, we have

$$
\begin{aligned}
c_{n}(X, Y)= & \frac{1}{2}\left(c_{n}(X, Y)-c_{n}(-Y,-X)\right) \\
= & \frac{1}{2}\left(\left[X, A_{n}(X, Y)\right]+\left[Y, B_{n}(X, Y)\right]\right. \\
& \left.-\left[-Y, A_{n}(-Y,-X)\right]-\left[-X, B_{n}(-Y,-X)\right]\right) .
\end{aligned}
$$

Therefore

$$
L_{n}(X, Y)=\frac{1}{2}\left(\left[X, A_{n}(X, Y)\right]+\left[Y, B_{n}(X, Y)\right]\right)
$$

satisfies (3.4).

Note that $L_{n}$ 's are not uniquely determined, however, we fix $L_{n}$ 's so that there holds

$$
L(X, v Y)=\left(\frac{e^{-\operatorname{ad} X}-1+\operatorname{ad} X}{\operatorname{ad} X\left(1-e^{-\operatorname{ad} X}\right)}-\frac{1}{4}\right) v Y+O\left(v^{2}\right),
$$

where we put $L(X, Y)=\sum_{n \geqq 2} L_{n}(X, Y)$ for $X, Y \in \mathscr{F}$ gl such that $X_{0}, Y_{0} \in$ $\mathfrak{g l}(N+2, \mathfrak{m})$. Thus, we obtain

$$
C(X, Y)=X+Y+[X, L(X, Y)]+[Y, L(-Y,-X)] .
$$

For a series $f=\sum_{n \in \mathbb{Z}} f_{n} t^{n} \in R\left[\left[t, t^{-1}\right]\right]$, we write

$$
\operatorname{Res}_{t} f=f_{-1} \in R \text {. }
$$

Let $R_{0}=\mathbb{R}[[z, \rho]] \subset R$, the formal power series in $z$ and $\rho$ over $\mathbb{R}$. We define a $R_{0}$-valued 2-cocycle $\omega$ on $\mathscr{F} \mathrm{gl}$ by

$$
\omega(X, Y)=\operatorname{Res}_{t} B\left(X, \partial_{t} Y\right)
$$

for $X, Y \in \mathscr{F}$ gl. Note that

$$
\omega\left(X^{*}, Y^{*}\right)=-\omega(X, Y)
$$

for $X, Y \in \mathscr{F} \mathrm{gl}$.

Now we introduce a group 2-cocycle on $\mathscr{F} \mathscr{G}_{0}$, following [BM]. Note that, from (3.3), any element $g \in \mathscr{F} \mathscr{G}_{0}$ can be uniquely written as $g=e^{X}$ for $X \in \mathscr{F}$ gl with $X_{0} \in \mathfrak{g l}(N+2, \mathfrak{m})$.

Definition. Let $\Xi$ be a $R_{0}$-valued function on $\mathscr{F} \mathscr{G}_{0} \times \mathscr{F} \mathscr{G}_{0}$ defined by

$$
\Xi\left(e^{X}, e^{Y}\right)=\omega(X, L(X, Y))+\omega(Y, L(-Y,-X)) .
$$


Then $\Xi$ defines a 2 -cocycle on $\mathscr{F} \mathscr{G}_{0}$, i.e. satisfies the cocycle condition:

$$
\Xi\left(e^{X}, e^{Y}\right)+\Xi\left(e^{X} e^{Y}, e^{Z}\right)=\Xi\left(e^{Y}, e^{Z}\right)+\Xi\left(e^{X}, e^{Y} e^{Z}\right)
$$

for $X, Y, Z \in \mathscr{F} \mathrm{gl}$.

Now we collect some basic properties of $\Xi$. For details we refer to [BM, S]. It follows from (3.6) that

$$
\Xi\left(\theta^{(\infty)} g_{1}, \theta^{(\infty)} g_{2}\right)=-\Xi\left(g_{1}, g_{2}\right)
$$

for $g_{1}, g_{2} \in \mathscr{F} \mathscr{G}_{0}$. In addition, $\Xi$ satisfies the anti-symmetric conditions:

$$
\begin{aligned}
\Xi\left(e^{X}, e^{Y}\right) & =-\Xi\left(e^{-X}, e^{X} e^{Y}\right) \\
& =-\Xi\left(e^{X} e^{Y}, e^{-Y}\right) \\
& =-\Xi\left(e^{-Y}, e^{-X}\right) .
\end{aligned}
$$

Define the mixed form $\Xi^{\prime}$ of $\Xi$ by

$$
\Xi^{\prime}\left(e^{X}, Y\right)=\left.\frac{d}{d v}\right|_{v=0} \Xi\left(e^{X}, e^{v Y}\right) .
$$

Then, using (3.7), (3.9) and the formula $e^{-X} \partial e^{X}=\frac{1-e^{-\operatorname{ad} X}}{\operatorname{ad} X} \partial X$, we obtain

$$
\begin{aligned}
\partial \Xi\left(e^{X}, e^{Y}\right)= & \Xi^{\prime}\left(e^{-Y} e^{-X}, \partial e^{X} e^{-X}\right)-\Xi^{\prime}\left(e^{-X}, \partial e^{X} e^{-X}\right) \\
& +\Xi^{\prime}\left(e^{X} e^{Y}, e^{-Y} \partial e^{Y}\right)-\Xi^{\prime}\left(e^{Y}, e^{-Y} \partial e^{Y}\right),
\end{aligned}
$$

where $\partial$ denotes either $\partial_{z}$ or $\partial_{\rho}$,

Lemma 3.2. $\Xi$ is trivial on $\mathscr{F} \mathscr{H} \times \mathscr{F} \mathscr{H}$, i.e.

$$
\Xi\left(g_{1}, g_{2}\right)=0 \text { for all } g_{1}, g_{2} \in \mathscr{F} \mathscr{H} \text {. }
$$

For proof, we refer to [S].

\section{Central Extension}

For any $p \in \mathscr{S} \mathscr{P}$, we can find an element $g \in \mathscr{F} \mathscr{H}$ which sends the identity element $1 \in \mathscr{S} \mathscr{P}$ to $p$ by Corollary 2.2. Then we have $p=k g$ for some $k \in \mathscr{F} \mathscr{K}$.

Lemma 4.1. For $p \in \mathscr{S} \mathscr{P}$, let $g \in \mathscr{F} \mathscr{H}$ be as above. If we put

$$
\partial_{z} p p^{-1}=\sum_{n \geqq 0} q_{n} t^{n}
$$

and

$$
p \cdot g^{-1} \partial_{z} g \cdot p^{-1}=\sum_{n \in \mathbb{Z}} a_{n} t^{n}
$$

then we have

$$
a_{0}+a_{0}^{*}=q_{0}+q_{0}^{*}
$$

and

$$
a_{n}+(-1)^{n} a_{-n}^{*}=q_{n}
$$

for $n=1,2,3, \ldots$ 
Proof. By the choice of $g$, we have

$$
m=g^{*} g=p^{*} p
$$

which imply that

$$
\begin{aligned}
m^{-1} \partial_{z} m & =m^{-1}\left(g^{-1} \partial_{z} g\right)^{*} m+g^{-1} \partial_{z} g \\
& =m^{-1}\left(p^{-1} \partial_{z} p\right)^{*} m+p^{-1} \partial_{z} p
\end{aligned}
$$

Therefore we obtain

$$
p^{-1}\left(p g^{-1} \partial_{z} g p^{-1}\right)^{*} p+g^{-1} \partial_{z} g=p^{-1}\left(\partial_{z} p p^{-1}\right)^{*} p+p^{-1} \partial_{z} p .
$$

Multiplying (4.3) by $p$ to the left and by $p^{-1}$ to the right, we have

$$
\left(p g^{-1} \partial_{z} g p^{-1}\right)^{*}+p g^{-1} \partial_{z} g p^{-1}=\left(\partial_{z} p p^{-1}\right)^{*}+\partial_{z} p p^{-1} \text {. }
$$

Hence

$$
\sum_{n \in \mathbb{Z}}\left\{a_{n}+(-1)^{n} a_{-n}^{*}\right\} t^{n}=\sum_{n>0} q_{n} t^{n}+q_{0}+q_{0}^{*}+\sum_{n<0}(-1)^{n} q_{-n}^{*} t^{n} .
$$

Comparing the coefficient of $t^{n}$ in both sides, we obtain (4.1) and (4.2).

Proposition 4.2. For $p \in \mathscr{S} \mathscr{P}$, let $g \in \mathscr{F} \mathscr{H}$ and $k \in \mathscr{F} \mathscr{K}$ such that $p=k g$. Let $\Xi$ be the 2-cocycle on $\mathscr{F} \mathscr{G}_{0}$ given in Sect. 3. Then we have the following identities:

$$
\begin{aligned}
& \partial_{z} \Xi\left(k g, g^{-1}\right) \\
& \quad=\frac{\rho}{2} \operatorname{Res}_{t} B\left(\frac{1}{2}\left(1+\frac{1}{t^{2}}\right) \mathscr{A}_{z}+\frac{1}{2}\left(-1+\frac{1}{t^{2}}\right) \mathscr{I}_{z}+\frac{1}{t} \mathscr{I}_{\rho}, p g^{-1} \partial_{z} g p^{-1}\right) \\
& \partial_{\rho} \Xi\left(k g, g^{-1}\right) \\
& =\frac{\rho}{2} \operatorname{Res}_{t} B\left(\frac{1}{2}\left(1+\frac{1}{t^{2}}\right) \mathscr{A}_{\rho}+\frac{1}{2}\left(-1+\frac{1}{t^{2}}\right) \mathscr{I}_{\rho}-\frac{1}{t} \mathscr{I}_{z}, p g^{-1} \partial_{z} g p^{-1}\right) .
\end{aligned}
$$

Proof. We shall use the following identity:

$$
\partial \Xi\left(k g, g^{-1}\right)=\frac{1}{2} \operatorname{Res}_{t}\left\{B\left(p^{-1} \partial_{t} p, g^{-1} \partial g\right)-B\left(p^{-1} \partial p, g^{-1} \partial_{t} g\right)\right\}
$$

which follows from (3.5) and (3.10), where $\partial$ denotes either $\partial_{z}$ or $\partial_{\rho}$.

Since $p \in \mathscr{S} \mathscr{P}$, we have

$$
p^{-1} \partial_{t} p+\frac{\rho\left(1+t^{2}\right)}{t\left(1-t^{2}\right)} p^{-1} \partial_{\rho} p=\frac{\rho\left(1+t^{2}\right)}{t\left(1-t^{2}\right)} p^{-1}\left\{\mathscr{A}_{\rho}+\frac{1-t^{2}}{1+t^{2}} \mathscr{I}_{\rho}-\frac{2 t}{1+t^{2}} \mathscr{I}_{z}\right\} p
$$

On the other hand, by Lemma 2.1, we have

$$
g^{-1} \partial_{t} g=-\frac{\rho\left(1+t^{2}\right)}{t\left(1-t^{2}\right)} g^{-1} \partial_{\rho} g
$$


From (4.6) with $\partial=\partial_{\rho},(4.7)$ and (4.8), we obtain

$$
\begin{aligned}
\partial_{\rho} \Xi\left(k g, g^{-1}\right) & =\frac{1}{2} \operatorname{Res}_{t} \frac{\rho\left(1+t^{2}\right)}{t\left(1-t^{2}\right)} B\left(\mathscr{A}_{\rho}+\frac{1-t^{2}}{1+t^{2}} \mathscr{I}_{\rho}-\frac{2 t}{1+t^{2}} \mathscr{I}_{z}, p g^{-1} \partial_{\rho} g p^{-1}\right) \\
& =\frac{1}{2} \operatorname{Res}_{t} \frac{\rho\left(1+t^{2}\right)}{t\left(1-t^{2}\right)} B\left(\mathscr{A}_{\rho}+\frac{1-t^{2}}{1+t^{2}} \mathscr{I}_{\rho}-\frac{2 t}{1+t^{2}} \mathscr{I}_{z}, \frac{1-t^{2}}{2 t} p g^{-1} \partial_{z} g p^{-1}\right) \\
& =\text { (the right-hand side of }(4.5)),
\end{aligned}
$$

where we used $\partial_{\rho} g=\frac{1-t^{2}}{2 t} \partial_{z} g$. Similarly, we can prove (4.4).

By (1.1), each element $X \in \mathfrak{g}$ decomposes uniquely as $X=X_{1}+X_{2}$ with $X_{1} \in \mathfrak{f}_{R}$ and $X_{2} \in \mathfrak{p}_{R}$. We shall denote $X_{1}$ and $X_{2}$ by $(X)_{\mathfrak{f}}$ and $(X)_{\mathfrak{p}}$, respectively.

Now we can prove the following proposition.

Proposition 4.3. For $p=\sum_{n \geqq 0} p_{n} t^{n} \in \mathscr{S P}$, let $g \in \mathscr{F} \mathscr{H}$ and $k \in \mathscr{F} \mathscr{K}$ be such that $p=k g$. Let $\tau$ be a solution of (1.8) and (1.9) corresponding to $P=p_{0}$. Then we have the following relations:

$$
\begin{gathered}
\tau^{-1} \partial_{z} \tau=\partial_{z} \Xi\left(k g, g^{-1}\right), \\
\tau^{-1} \partial_{\rho} \tau=\partial_{\rho} \Xi\left(k g, g^{-1}\right)
\end{gathered}
$$

Proof. First, expanding (2.12.a) in a series of $t$, we have

$$
\begin{aligned}
\partial_{z} p p^{-1} & =\left(\mathscr{A}_{z}+\mathscr{I}_{z}\right)+2 \mathscr{I}_{\rho} t+O\left(t^{2}\right) \\
& =\sum_{n \geqq 0} q_{n} t^{n} .
\end{aligned}
$$

Thus, taking the coefficients of $t^{0}$ and $t^{1}$, we obtain

$$
q_{0}=\mathscr{A}_{z}+\mathscr{I}_{z} \text { and } q_{1}=2 \mathscr{I}_{\rho}
$$

In the right-hand side of (4.4), since $\mathfrak{f}_{R}$ and $\mathfrak{p}_{R}$ are orthogonal to each other with respect to $B$, we have

$$
\begin{aligned}
\operatorname{Res}_{t}\left(1+\frac{1}{t^{2}}\right) B\left(\mathscr{A}_{z}, p g^{-1} \partial_{z} g p^{-1}\right) & =B\left(\mathscr{A}_{z},\left(a_{1}+a_{-1}\right)_{\mathfrak{t}}\right) \\
\operatorname{Res}_{t}\left(-1+\frac{1}{t^{2}}\right) B\left(\mathscr{I}_{z}, p g^{-1} \partial_{z} g p^{-1}\right) & =B\left(\mathscr{I}_{z},\left(a_{1}-a_{-1}\right)_{\mathfrak{p}}\right) \\
\operatorname{Res}_{t} \frac{1}{t} B\left(\mathscr{I}_{\rho}, p g^{-1} \partial_{z} g p^{-1}\right) & =B\left(\mathscr{I}_{\rho},\left(a_{0}\right)_{\mathfrak{p}}\right)
\end{aligned}
$$


where we put $p g^{-1} \partial_{z} g p^{-1}=\sum_{n \in \mathbb{Z}} a_{n} t^{n}$. Then, by Lemma 4.1 and (4.11), we have

$$
\begin{aligned}
\left(a_{1}+a_{-1}\right)_{\mathfrak{f}} & =\frac{1}{2}\left(a_{1}+a_{-1}-a_{1}^{*}-a_{-1}^{*}\right) \\
& =\frac{1}{2}\left(q_{1}-q_{1}^{*}\right)=0 \\
\left(a_{1}-a_{-1}\right)_{\mathfrak{p}} & =\frac{1}{2}\left(a_{1}-a_{-1}+a_{1}^{*}-a_{-1}^{*}\right) \\
& =\frac{1}{2}\left(q_{1}+q_{1}^{*}\right)=2 \mathscr{I}_{\rho} \\
\left(a_{0}\right)_{\mathfrak{p}}= & \frac{1}{2}\left(a_{0}+a_{0}^{*}\right) \\
& =\frac{1}{2}\left(q_{0}+q_{0}^{*}\right)=2 \mathscr{I}_{z} .
\end{aligned}
$$

Therefore, recalling (1.8), we see that (4.9) holds.

Similarly, we can prove (4.10). This completes the proof.

Now we define a central extension of $\mathscr{F} \mathscr{G}_{0}$ in terms of the cocycle $\Xi$.

Definition. Let $\left(\mathscr{F} \mathscr{G}_{0}\right)^{\sim}$ be the set given by

$$
\left(\mathscr{F} \mathscr{G}_{0}\right)^{\sim}=\left\{\left(g, e^{\mu}\right) ; g \in \mathscr{F} \mathscr{G}_{0}, \mu \in R_{0}\right\} .
$$

Define a product of any two elements of $\left(\mathscr{F} \mathscr{G}_{0}\right)^{\sim}$ by

$$
\left(g_{1}, e^{\mu_{1}}\right) \cdot\left(g_{2}, e^{\mu_{2}}\right)=\left(g_{1} g_{2}, e^{\mu_{1}+\mu_{2}+\Xi\left(g_{1}, g_{2}\right)}\right)
$$

for $\left(g_{1}, e^{\mu_{1}}\right),\left(g_{2}, e^{\mu_{2}}\right) \in\left(\mathscr{F} \mathscr{G}_{0}\right)^{\sim}$. Since $\Xi$ satisfies the cocycle condition (3.7), $\left(\mathscr{F} \mathscr{G}_{0}\right)^{\sim}$ forms a group with group multiplication given by (4.12). Namely, $\left(\mathscr{F} \mathscr{G}_{0}\right)^{\sim}$ is a central extension of $\mathscr{F} \mathscr{G}_{0}$.

Let $\tilde{\theta}^{(\infty)}$ be an involution of $\left(\mathscr{F} \mathscr{G}_{0}\right)^{\sim}$ given by

$$
\tilde{\theta}^{(\infty)}\left(g, e^{\mu}\right)=\left(\theta^{(\infty)}(g), e^{-\mu}\right) .
$$

If we denote by $(\mathscr{F} \mathscr{K})^{\sim}$ the subgroup of $\left(\mathscr{F} \mathscr{G}_{0}\right)^{\sim}$ consisting of elements which are fixed by $\tilde{\theta}^{(\infty)}$, then we have

$$
(\mathscr{F} \mathscr{K})^{\sim}=\left\{(k, 1) \in\left(\mathscr{F} \mathscr{G}_{0}\right)^{\sim} ; k \in \mathscr{F} \mathscr{K}\right\} .
$$

Let $(\mathscr{F} \mathscr{P})^{\sim}$ be a subgroup of $\left(\mathscr{F} \mathscr{G}_{0}\right)^{\sim}$ given by

$$
(\mathscr{F} \mathscr{P})^{\sim}=\left\{\left(p, e^{\mu}\right) \in\left(\mathscr{F} \mathscr{G}_{0}\right)^{\sim} ; p \in \mathscr{F} \mathscr{P}, \mu \in R_{0}\right\} .
$$

It follows immediately from the decomposition (2.8) of $\mathscr{F} \mathscr{G}$ that $\left(\mathscr{F} \mathscr{G}_{0}\right)^{\sim}$ has a unique decomposition:

$$
\left(\mathscr{F} \mathscr{G}_{0}\right)^{\sim}=(\mathscr{F} \mathscr{K})^{\sim} \cdot(\mathscr{F} \mathscr{P})^{\sim} .
$$

Furthermore, we put

$$
(\mathscr{F} \mathscr{H})^{\sim}=\left\{\left(g, e^{\gamma}\right) \in\left(\mathscr{F} \mathscr{G}_{0}\right)^{\sim} ; g \in \mathscr{F} \mathscr{H}, \gamma \in \mathbb{R}\right\} .
$$


It follows from Lemma 3.2 that $\mathscr{F} \mathscr{H}$ can be regarded as a subgroup of $(\mathscr{F} \mathscr{H})^{\sim}$ by

$$
\mathscr{F} \mathscr{H} \rightarrow(\mathscr{F} \mathscr{H})^{\sim}, \quad g \mapsto(g, 1) .
$$

Let $(\mathscr{S} \mathscr{P})^{\sim}$ be the subset of $(\mathscr{F} \mathscr{P})^{\sim}$ given by

$$
\begin{aligned}
(\mathscr{S P P})^{\sim}=\left\{\left(p, e^{\mu}\right) \in(\mathscr{F} \mathscr{P})^{\sim} ; p=\sum_{n \geqq 0} p_{n} t^{n} \in \mathscr{S} \mathscr{P},\right. \\
\left.\tau=e^{-\mu} \text { satisfies (1.8) and (1.9) with } P=p_{0}\right\} .
\end{aligned}
$$

We call $(\mathscr{S} \mathscr{P})^{\sim}$ the space of potentials with conformal factor.

Proposition 4.4. For $p \in \mathscr{S} \mathscr{P}$, let $k \in \mathscr{F} \mathscr{K}$ and $g \in \mathscr{F} \mathscr{H}$ be as above, i.e. $p=k g$. Then we have

$$
\Xi\left(p^{*}, p\right)=2 \Xi\left(k g, g^{-1}\right) .
$$

Therefore, any element of $(\mathscr{S P P})^{\sim}$ can be written as $\left(p, e^{-\frac{1}{2} \Xi\left(p^{*}, p\right)+\gamma}\right)$ for $p \in \mathscr{S} \mathscr{P}$, $\gamma \in \mathbb{R}$.

Proof. Using the anti-symmetric condition (3.9) and the cocycle condition (3.7), we have

$$
\begin{aligned}
\Xi\left(p^{*}, p\right) & =-\Xi\left(p^{-1}, \theta^{(\infty)} p\right)=-\Xi\left(p^{-1}, k \theta^{(\infty)} g\right) \\
& =-\Xi\left(p^{-1}, k\right)-\Xi\left(p^{-1} k, \theta^{(\infty)} g\right)+\Xi\left(k, \theta^{(\infty)} g\right) .
\end{aligned}
$$

For the first and the last terms in the r.h.s. of (4.16), it follows from (3.9) and (3.8) that

$$
\Xi\left(p^{-1}, k\right)=-\Xi\left(k, \theta^{(\infty)} g\right)=\Xi(k, g) .
$$

The middle term in the r.h.s. of (4.16) vanishes by Lemma 3.2.

Now using (3.9) again, we obtain

$$
\begin{aligned}
\Xi(k, g) & =\Xi\left(p g^{-1}, g\right)=-\Xi\left(g^{-1}, g p^{-1}\right) \\
& =\Xi\left(g, p^{-1}\right)=-\Xi\left(p, g^{-1}\right) .
\end{aligned}
$$

Thus (4.15) follows.

For any $\left(p, e^{\mu}\right) \in(\mathscr{S} \mathscr{P})^{\sim}$, put $p=\sum_{n \geq 0} p_{n} t^{n}$. By definition and Proposition 4.3, both $\tau=e^{-\mu}$ and $\tau^{\prime}=e^{\frac{1}{2} \Xi\left(p^{*}, p\right)}$ satisfies (1.8) and (1.9) with $P=p_{0}$. Therefore they must be equal to each other, up to constant multiple. This completes the proof of the proposition.

Define an action of $(\mathscr{F} \mathscr{H})^{\sim}$ on the space of potentials with conformal factor $(\mathscr{S P})^{\sim}$ to the right through the decomposition (4.13):

$$
(\mathscr{S P})^{\sim} \times(\mathscr{F} \mathscr{H})^{\sim} \rightarrow(\mathscr{S} \mathscr{P})^{\sim},\left(\left(p, e^{\mu}\right),\left(g, e^{\gamma}\right)\right) \mapsto\left(p_{g}, e^{\alpha}\right) .
$$

Namely, we can find a unique element $(k, 1) \in(\mathscr{F} \mathscr{K})^{\sim}$ and $\left(p_{g}, e^{\alpha}\right) \in(\mathscr{F} \mathscr{P})^{\sim}$ such that

$$
\left(p, e^{\mu}\right)\left(g, e^{\gamma}\right)=(k, 1)^{-1}\left(p_{g}, e^{\alpha}\right),
$$

where $k$ and $p_{g}$ are the elements given in (2.19). Since we have

$$
\tilde{\theta}^{(\infty)}\left(\left(p, e^{\mu}\right)\left(g, e^{\gamma}\right)\right)^{-1} \cdot\left(p, e^{\mu}\right)\left(g, e^{\gamma}\right)=\left(g^{*} p^{*} p g, e^{2(\mu+\gamma)+\Xi\left(p^{*}, p\right)}\right)
$$


and

$$
\tilde{\theta}^{(\infty)}\left(p_{g}, e^{\alpha}\right)^{-1} \cdot\left(p_{g}, e^{\alpha}\right)=\left(p_{g}^{*} p_{g}, e^{2 \alpha+\Xi\left(p_{g}^{*}, p_{g}\right)}\right)
$$

we obtain

$$
\begin{aligned}
\alpha & =\mu+\gamma+\frac{1}{2}\left(\Xi\left(p^{*}, p\right)-\Xi\left(p_{g}^{*}, p_{g}\right)\right) \\
& =\gamma^{\prime}-\frac{1}{2} \Xi\left(p_{g}^{*}, p_{g}\right)
\end{aligned}
$$

for some $\gamma^{\prime} \in \mathbb{R}$, where we used Proposition 4.4. Thus $\left(p_{g}, e^{\alpha}\right)$ belongs to $(\mathscr{S} \mathscr{P})^{\sim}$, i.e. the action (4.18) of $(\mathscr{F} \mathscr{H})^{\sim}$ is well-defined.

Now we state our main theorem:

Theorem 4.5. The group $(\mathscr{F} \mathscr{H})^{\sim}$ acts transitively on the space of potentials with conformal factor $(\mathscr{S} \mathscr{P})^{\sim}$ by (4.18).

Proof. What remains to be proved is transitivity of the action. By Proposition 4.4, any element of $(\mathscr{S} \mathscr{P})^{\sim}$ can be written as $\left(p, e^{-\frac{1}{2} E\left(p^{*} p\right)+\gamma}\right)$. Moreover, by Corollary 2.3, $p \in \mathscr{S} \mathscr{P}$ can be written as $p=k g$ for $k \in \mathscr{F} \mathscr{K}$ and $g \in \mathscr{F} \mathscr{H}$. Then we have, by (4.17),

$$
\begin{aligned}
(k, 1)\left(g, e^{\gamma}\right) & =\left(k g, e^{\gamma+\Xi(k, g)}\right) \\
& =\left(p, e^{\gamma-\frac{1}{2} \Xi\left(p^{*}, p\right)}\right) .
\end{aligned}
$$

This shows that any element of $(\mathscr{S P P})^{\sim}$ is on the oribit of the identity element $(1,1) \in(\mathscr{S} \mathscr{P})^{\sim}$.

\section{References}

[BM] Breitenlohner, P., Maison, D.: On the Geroch group. Ann. Inst. Henri Poincare 46, 215-246 (1987)

[BMG] Breitenlohner, P., Maison, D., Gibbons, G: 4-Dimensional black holes from Kaluza-Klein theories. Commun. Math. Phys. 120, 295-333 (1988).

[DO] Doi, H., Okamoto, K.: A survey of the generalized Geroch conjecture. Adv. Stud. in Pure Math. 14, 379-393 (1988)

[E] Ernst, F.J.: New formulation of the axially symmetric gravitational field problem II. Phys. Rev. 168, 1415-1417 (1968)

[G] Geroch, R.: A method for generating new solutions of Einstein's equations II. J. Math. Phys. 13, 394-404 (1972)

[GX] Gürses, M., Xanthopoulos, B.C.: Axially symmetric, static self-dual SU(3) gauge fields and stationary Einstein-Maxwell metrics. Phys. Rev. D 26, 1912-1915 (1982)

[HS] Hashimoto, T., Sawae, R.: A linearization of $S(U(1) \times U(2)) \backslash S U(\mathbf{1}, 2) \sigma$-model. Hiroshima Math. J. 22, 445-459 (1992)

[HE] Hauser, I., Ernst, F.J.: Proof of a generalized Geroch conjecture. In: Galaxies axisymmetric systems and relativity, M.A.H. MacCallum (ed.). Cambridge: Cambridge Univ. Press 1985, p. 115

[K] Kac, V.G.: Infinite dimensional Lie algebras. Boston: Birkhäuser 1983

[Ki] Kinnersley, W.: Symmetries of the stationary Einstein-Maxwell field equations I. J. Math. Phys. 18, 1529-1537 (1977)

[Kn] Knapp, A.W.: Representation theory of semisimple groups - An overview based on examples. Princeton: Princeton Univ. Press 1986 
[N] Nagatomo, K.: The Ernst equation as a motion on a universal Grassmann manifold. Commun. Math. Phys. 122, 423-453 (1989)

[S] Sawae, R.: The conformal factor and a central extension of a formal loop group with values in $\operatorname{PSL}(2, \mathbb{R})$ Hiroshima Math. J. (to appear)

[T] Takasaki, K.: A new approach to the self-dual Yang-Mills equations II. Saitama Math. J. 3, 11-40 (1985)

[V] Varadarajan, V.S.: Lie groups, Lie algebras, and their representations. Berlin, Heidelberg, New York: Springer 1984

[W] Weyl, H.: Zur Gravitationstheorie. Ann. der Phys. 54, 117-145 (1917)

Communicated by H. Araki 
